\title{
Real-Time Implementation of Nonlinear State-of-Charge Estimation Techniques for Hybrid Electric Vehicles
}

\author{
Roxana-Elena Tudoroiu ${ }^{1}$, Wilhelm Kecs ${ }^{2}$, Maria Dobritoiu ${ }^{3}$, Nicolae Ilias ${ }^{4}$, \\ Valentin-Stelian Casavela ${ }^{5}$, Dumitru Burdescu ${ }^{6}$ and Nicolae Tudoroiu ${ }^{* 7}$ \\ 1,2, 3, 4, 5 University of Petrosani, Petrosani, 332006, Romania. \\ ${ }^{6}$ University of Craiova, Craiova, 200585, Romania. \\ ${ }^{7}$ John Abbott College, Saint-Anne-de-Bellevue, QC H9X 3L9, Canada. \\ e-mail: ntudoroiu@gmail.com
}

\begin{abstract}
The problem of State-of-Charge (SOC) estimation is crucial in automotive industry for successful marketing of both electric vehicles (EVs) and hybrid electric vehicles (HEVs). Nowadays the wealth of rechargeable $\mathrm{Ni}-\mathrm{MH}$ and $\mathrm{Li}$-ion batteries is a great prospective to be used extensively to plug-in hybrid electric vehicles (PHEVs), hybrid electric vehicles (HEVs), and battery electric vehicles (BEVs). In addition the Li-ion batteries are much lighter in weight and of reduced size, therefore much easier to be integrated in the vehicle structure in order to provide more power to boost up the acceleration, and to increase the energy efficiency through on-board battery energy storage by means of regenerative braking and mechanical-electric energy conversion. Gradual capacity reduction and performance decay can be evaluated strictly based on the current knowledge of rechargeable battery technology, and consequently is required a rigorous monitoring and a tight control of the SOC level, that is essential for increasing the operating lifetime of the rechargeable batteries. In this paper we present some preliminary results obtained in our research to implement some of SOC estimation techniques for the rechargeable batteries of different chemistry. We investigate the effectiveness of two nonlinear Kalman Filters estimators (Extended and Unscented versions) in order to find the most suitable estimator, more accurate and to perform better. The contribution of this paper is the implementation of these estimators in real time based on a generic model of the battery by means of extensive simulations in a MATLAB/SIMULINK programming environment.
\end{abstract}

Key words: Hybrid Electric Vehicles, State of Charge, Extended Kalman Filter, Unscented Kalman Filter.

\section{Introduction}

The State-of-Charge (SOC) of a battery is its available capacity expressed as a percentage of its rated capacity. The problem of state-of-charge (SOC) estimation for both electric vehicles (EVs) and hybrid electric vehicles (HEVs) is of vital importance for accurate estimation of remaining battery capacity and prediction of the instantaneous battery power that can be delivered to the electric engine. Determination of an optimum battery pack management system (BMS) is perhaps the one of the most significant technical issue for the automotive industry to have a successful marketing of HEVs. More particularly, an optimal operating vehicle energy and battery management system (BMS) should have the following features based on an accurate SOC estimate and a precise available battery power forecast:

a) avoid overcharging and undercharging damages to the battery

b) aggressively use the entire battery pack capacity

c) enhance overall power system performance and its reliability

d) allow the use of smaller and lighter battery packs, and

e) reduced production and service related costs.

These issues are among the main motivating factors for the research proposed in this paper. The necessity of the rigorous consistency requirements envisaged for HEVs do indeed justify the 
development and implementation of more advanced estimation and prediction techniques for the optimal design of the BMSs. However due to the high complexity, nonlinearity, time-variation, and uncertain behavior of the batteries in HEVs, it is quite difficult to achieve reliable, accurate and satisfactory estimation/prediction performance specifications using model-based approaches that rely on linear models or linearized models of the batteries of different chemistry, such as techniques that are based on Kalman filtering (KF) Extended Kalman filtering (EKF) [1]. Thus, there is a need to develop nonlinear model-based estimation techniques $[1,2,3,4,5,6]$ that rely and are based on the true nonlinear model of the process.

Towards this objective, nonlinear model-based techniques have been extensively studied in the literature. In this paper, in addition to the EKF technique, which has already been developed in [1] for SOC estimation in Li-PB batteries, we have developed and implemented a new extension for nonlinear estimation techniques including Unscented Kalman Filter (UKF) for SOC estimation of nickel metal hydride (Ni-MH) batteries. The remainder of the paper is organized as follows. In section 2, the generic Ni-MH battery modeling issues are introduced together with the other batteries modeling aspects. In section 3 are developed the both EKF and UKF filtering nonlinear estimators based on the generic Ni-MH battery model chosen in section 2 in order to estimate the battery SOC. The simulation results for both nonlinear estimators in MATLAB/SIMULINK programming environment are given in section 4 . The robustness capability of the both estimators is also evaluated in the same section. Finally, performance comparisons of the both algorithms and conclusions are given in section 5 .

\section{Ni-MH Battery Model Description}

Battery modeling forms the basis of and stands as an useful tool for battery design, manufacturing, and control. It is particularly important for battery characterization (i.e., State-of-Charge (SOC) and State-of-Health (SOH) estimation) and battery management since the model development is logically the first step in developing any system identification and adaptive state estimation algorithms [4]. The most common models can be generally classified into two groups: electrochemical models and equivalent circuit models. Equivalent circuit models consisting of electrical circuit components such as capacitors, resistors, diodes, and voltage sources, can be readily developed using electric circuit simulation software such as PSpice [7]. Other types of models, given in algebraic or differential equations, may be more suitable for a generic simulation environment such as MATLAB/SIMULINK. Recently, MATLAB also released a generic battery model in its SimPower systems toolbox [7]. Nevertheless, an equivalent circuit model can be easily converted into other model formats. The choice of model representation will be determined by the matter of convenience and the simulation tools available.

National Renewable Energy Laboratory (NREL) also developed an electric circuit model for batteries that is basically a RC network, as shown in figure 1, and is a part of its ADVISOR tool package $[7,8]$.

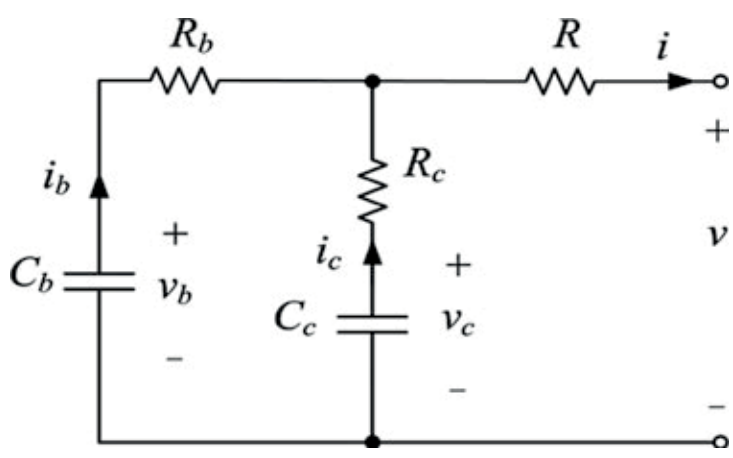

Figure 1. NREL battery model $[7,8]$

The model contains two capacitors $\left(C_{b}\right.$ and $\left.C_{c}\right)$ and three resistors $\left(R_{b}, R_{c}\right.$, and $\left.R\right)$. The capacitor $C_{b}$ models the main storage capacity of the battery. The capacitor $C_{c}$ captures the fast charge and discharge aspect of the battery and is much smaller than $C_{b}$ [7]. The model has a big advantage that can be converted into other model formats for the convenience of simulation. A full representation of the battery model in state space is described in detail in [7], and can be considered as a generic representation for all the batteries of different chemistry. In particular, the nickel-metal hydride (Ni-MH) batteries in a tight competition with LiIon batteries can be represented successfully by the same generic model with the battery thermal model represented by a lumped first-order equation with linear dynamics [7]. The parameters of the components are functions of the SOC and battery 
temperature (T). In addition, the resistance also depends on whether the battery is in "charge" or "discharge" mode. The overall SOC is a weighted combination of the states of charge on $C_{b}$ and $C_{c}$ [7]. Mathematical modeling is indispensable in this process since a cell model, once validated experimentally, can be used to identify celllimiting mechanisms and forecast the cell performance for design, scale-up, and optimization. The both algorithms developed in this paper are implemented based on a similar generic Ni-MH battery model. The various modeling aspects and phenomena inside the battery such as thermodynamics, polarizations, hysteresis, etc. have been incorporated into the battery model. Also, to improve the estimation performance, this Ni-MH battery model was combined with the coulomb-accumulation model developed in [7]. A very useful tool for performance evaluation of both nonlinear estimators for different standard driving cycles (current profiles) is provided by Advisor 3.2 software package.

\section{Prediction and Estimation Techniques}

The ultimate goal of state and parameter system identification is to obtain a mathematical model whose output matches the output of a dynamic process that is subjected to a given input. The solution to the exact matching problem, in general, is extremely difficult. Consequently, for practical reasons the original problem is commonly relaxed to developing a model whose output can be made "as close as possible" (in some metric sense) to the output of the dynamic system. Different methods have been developed in the literature for both linear and nonlinear system identifications and $[1$, $2,3,4,5,6]$. A common characteristic of most of these methods is the use of a parameterized model where the parameters are recursively updated in real-time to minimize a performance index such as the output identification error. These methods can be broadly classified into two main categories, namely conventional model-based schemes and intelligent and neural network-based schemes. Conventional methods are well established for linear systems and have recently been under investigation for generalization to nonlinear systems. The main disadvantage of these methods is that they are generally applicable and extendable to only a special and quite limited class of nonlinear systems. A good survey of conventional nonlinear identification methods may be found in [4].

\subsection{Extended Kalman Filter Estimator}

The Kalman filter is a powerful and popular tool for the stochastic estimation problem that is proposed by R.E. Kalman in 1960 [1]. Consider a linear stochastic difference equation:

$$
\begin{aligned}
x_{k+1} & =A x_{k}+B u_{k}+w_{k} \\
y_{k} & =C x_{k}+D u_{k}+v_{k}
\end{aligned}
$$

where $w_{k}$ and $v_{k}$ are the process and measurement noise, respectively, and are assumed to be independent white Gaussian random process with zero mean value,

$E\left[w_{n} w_{n}^{T}\right]=\left\{\begin{array}{c}\sum_{w}, n=k \\ 0, n \neq k\end{array}, E\left[v_{n} v_{n}^{T}\right]=\left\{\begin{array}{c}\sum_{v}, n=k \\ 0, n \equiv k\end{array}\right.\right.$

The distributions of the process and measurement noise are the normal probability distributions

$$
p(w) \sim N\left(0, Q_{w}\right), \quad p(v) \sim N\left(0, R_{v}\right)
$$

The covariance matrices $Q_{w}$ (process noise covariance) and $R_{v}$ (measurement noise covariance) might change with each time step or measurement, but in our approach we assume they are constant. Due to the process noise injected in the state equation (1), the state vector $x_{k} \in R^{n}$ becomes random variable with its distribution approximated by a Gaussian distribution function:

$$
p(x) \sim G\left(\hat{x}, P_{x}\right)
$$

By applying a predictor-corrector algorithm, the Kalman filter estimates the process state at current time and then obtains feedback in the form of the noisy measurements. As such, the process of the filter falls into two steps, namely time update and measurement update. During the time update, the current state and error covariance estimate are projected forward (in time) to obtain a priori estimate of the state in the next time step. Next, the new measurement is incorporated into this priori estimate value for calculating the posteriori estimate of the corresponding state during the measurement update $[1,2,3,4,5,6]$. However, the process to be estimated and the measurement 
relationship to the process can be nonlinear in practice. Under these situations the most interesting and successful applications of the Kalman filter is the use of an underlying linearized process model whereby an estimate of the current state of the system is made and then its estimate is corrected by using any available sensor or output measurements. Following the same predictor-corrector mechanism, it is then possible to approximately obtain an optimal state estimate of the state and its covariance. This linearized extension of Kalman filter is referred to as the Extended Kalman filter or $\operatorname{EKF}[1,2,3,4]$.

\subsection{Unscented Kalman Filter Estimator}

The Unscented Kalman Filter (UKF) technique $[2,3,4,5,6]$ is based on the unscented transformation (UT) and addresses the general problem of state estimation $x_{k} \in R^{n}$. The discretetime process is governed by a nonlinear stochastic difference state-space equation with a measurement $y_{k} \in R^{m}$ and is given by

$$
\begin{aligned}
& x_{k+1}=f\left(x_{k}, u_{k}\right)+w_{k} \\
& y_{k}=g\left(x_{k}, u_{k}\right)+v_{k}
\end{aligned}
$$

The random variables $w_{k}$ and $v_{k}$ are representing the process and measurement noise, respectively. They are assumed to be Gaussian, mutually independent, zero mean, white, and with normal probability distributions:

$$
p(w) \sim N\left(0, Q_{w}\right), \quad p(v) \sim N\left(0, R_{v}\right)
$$

The covariance matrices $Q_{w}$ (process noise covariance) and $R_{v}$ (measurement noise covariance) might change with each time step or measurement, but in our approach we assume they are constant. Due to the process noise injected in the state space equation the state vector $x_{k} \in R^{n}$ becomes random variable with its distribution approximated by a Gaussian distribution function $p(x) \sim G\left(\hat{x}, P_{x}\right)$. The vital operation performed in the Kalman Filter is the propagation of a Gaussian random state variable $x_{k} \in R^{n}$ through the system dynamics. In the
Extended Kalman Filter (EKF) the Gaussian random state variable $x_{k} \in R^{n}$ is propagated analytically through the first-order linearization of the nonlinear system. This can introduce large errors in the true posterior mean and covariance of the transformed Gaussian random state variable, which may lead to sub-optimal performance and sometimes divergence of the filter. The UKF approach is developed as an alternative to the EKF and addresses this problem by using a deterministic sampling approach. Using the principle that a minimal set of carefully chosen sample points can be used to parameterize mean and covariance, the UKF yields superior performance compared to EKF, especially for nonlinear systems. These sample points completely capture the true mean and covariance of the Gaussian random state variable $x_{k} \in R^{n}$, and are propagated through the true nonlinear system dynamics. The cloud of the transformed sample points' distribution captures the posterior mean and covariance accurately to the fourth order while the EKF only predicts with accuracy up to the second order for the posterior mean and fourth order for the covariance $[2,5,6]$. However, the UKF will make more accurate estimates only if the kurtosis and higher order moments in the state error distribution are significant. In some applications the sampling rate could be an important source of degrading in the UKF performance. The main advantage of the UKF is that it does not require the calculation of the Jacobian matrices that could lead to implementation difficulties $[2,5,6]$.

\subsection{Real-Time Implementation of Extended and Unscented Kalman Filters Estimators}

Headings Extensive simulation results for the SOC estimation using the EKF, the UKF, and the RHNF algorithms are explained and compared in this section. Comparative analysis of all the three proposed algorithms in terms of the steady state performance and the robustness capability are also presented in details.

First, the performance evaluation under the nominal battery parameters, namely the battery internal resistance $R_{i}$ and the battery capacity $C_{n}$ is presented and then the robustness capability of the algorithms with respect to large initial SOC errors 
and changes in the battery parameters is illustrated also. The simulations are carried out using the reference driving cycle shown in Figure 2.

\subsection{Simulation Results in MATLAB Programming Environment}

The simulation results in MATLAB programming environment for the both algorithms for the nominal battery parameters and different levels of uncertainty in the initial value of SOC are given in the Figures 2-6.

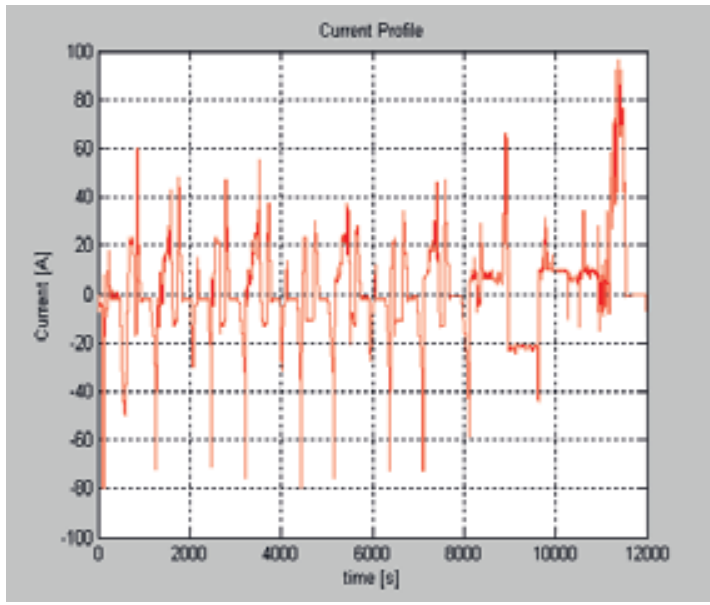

Figure 2. The current profile used in simulations

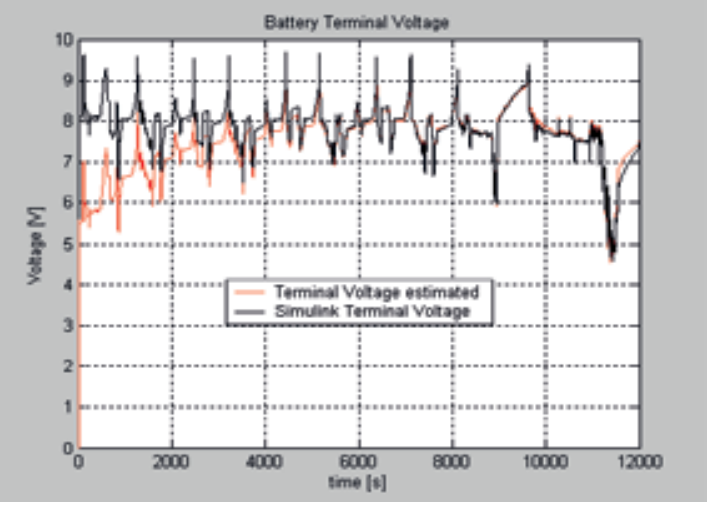

Figure 3. The Battery Terminal Voltage Profile

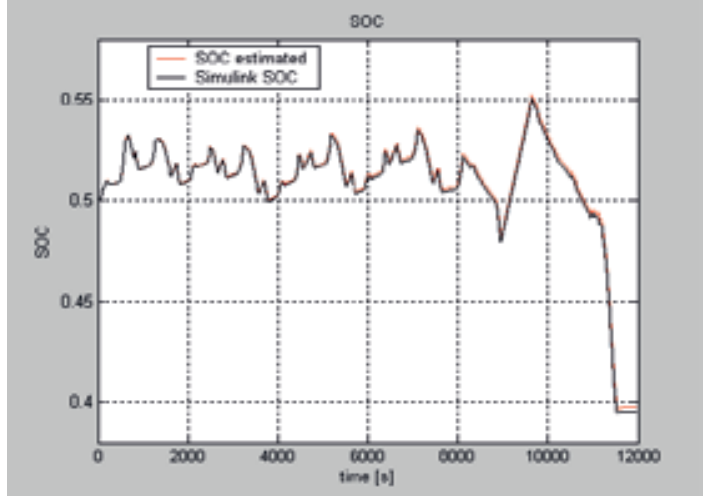

Figure 4. The Battery Terminal Voltage Profile

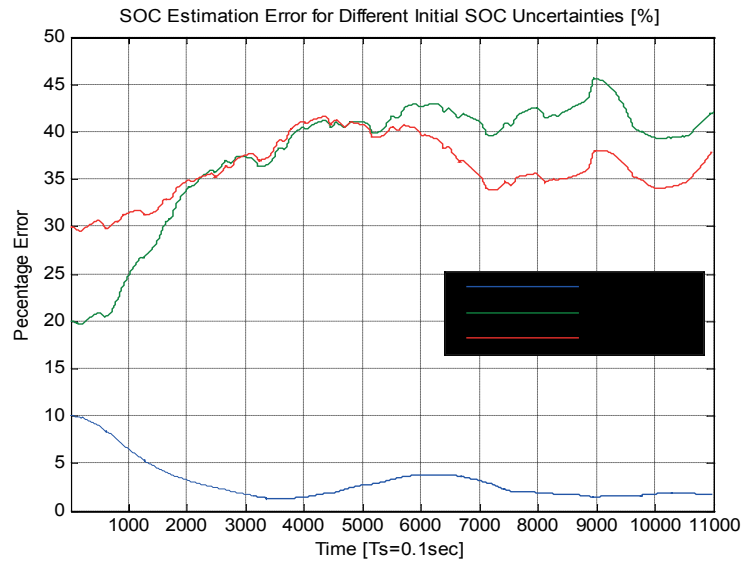

Figure 5. The SOC estimation error using the EKF Estimator

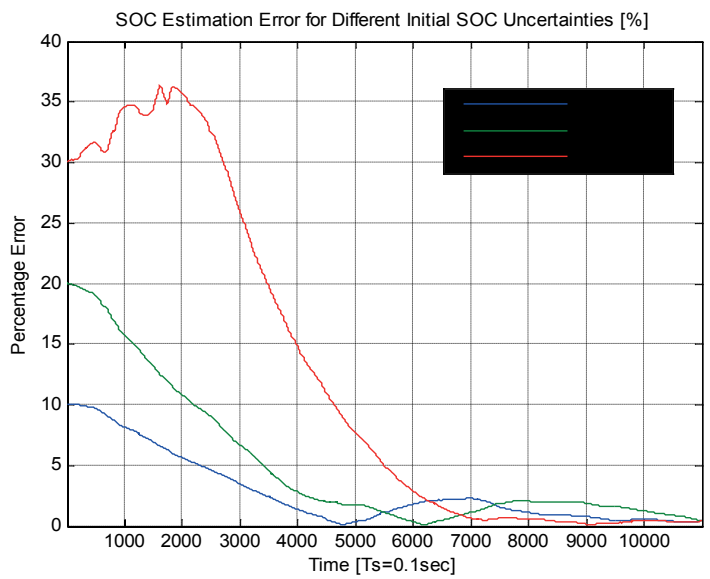

Figure 6. The SOC estimation error using the UKF Estimator 
The robustness of the both estimators to the changes in the battery parameters (Resistance and capacitance) is depicted in the Figures 8-9.

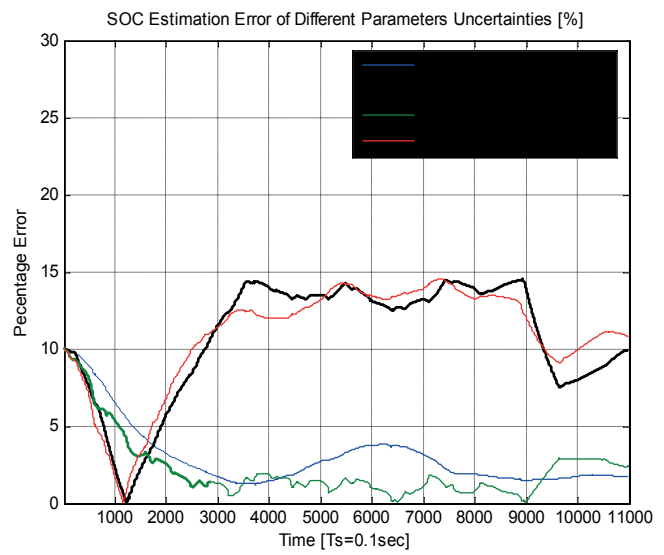

Figure 7. The SOC estimation error using the EKF Estimator

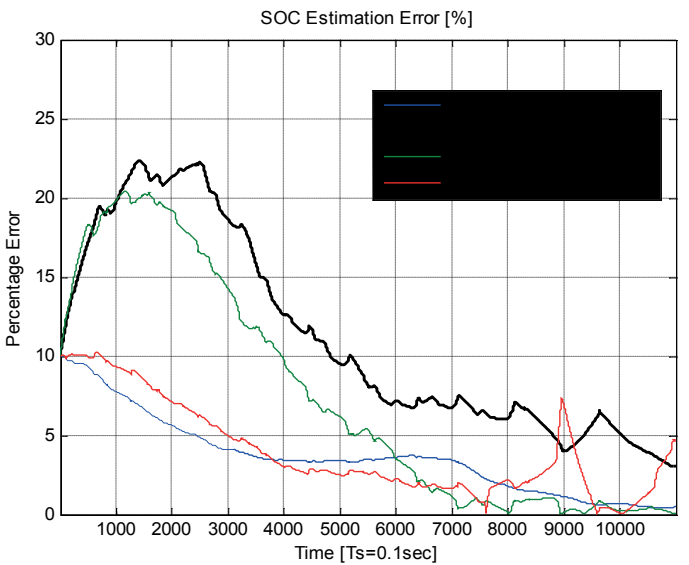

Figure 8. The SOC estimation error using the UKF Estimator

\section{Conclusions}

For an overall comparative performance of the both algorithms in a more comprehensible framework, the results developed are summarized as follows:

1) The EKF algorithm performs well only for some of the driving cycles

2) The UKF algorithm performs very well for almost all the driving cycles with standard steady state errors sometimes smaller than $2 \%$.
3) The UKF algorithm outperforms the EKF algorithm for almost all the driving cycles in terms of the convergence and the steady state error.

4) The EKF algorithm is not convergent in nearly all cases

5) The UKF algorithm converges very well for majority of the driving cycles

6) The EKF algorithm is not robust in almost all conditions

7) The UKF algorithm is robust in the steady state while it shows a large error in the transient phase

\section{References}

1) Plett, G., Journal of Power Source, 134 (2), (2004), 252.

2) LaViola, J.J.Jr, Proceedings of the American Control Conference, 2003, Denver, Colorado, June 4-June 6, 2003.

3) Wan, E. A., and Rudolph van der Merwe, Proceedings of Symposium on Adaptive Systems for Signal processing, Communication and Control (AS-SPCC) IEEE Press, 2000, Lake Louise, Canada, October 2000.

4) Simon Haykin, Adaptive filter theory fourth edition, edited by T. Kaillath (Prentice - Hall, NJ, 2003), 936.

5) Tudoroiu, N., Zaheerudin, M.,Cretu, V., and R-E., Tudoroiu, IEEE Industrial Electronics Magazine 4(3), (2010), 7.

6) Tudoroiu, N., Zaheerudin, M.,Chiru, C., Grigore, M., and R-E., Tudoroiu, HSI'09 Proceedings of the 2nd conference on Human System Interactions 2009, Catania, Italy, May 2009.

7) Kwo Young, Caisheng Wang, Le Yi Wang, and Kai Strunz, Power Electronics and Power Systems-Chapter 2, edited by Rodrigo GarciaValle, Joao A Peças Lopes (Springer, Germany, 2013), 325

8) Johnson V.H., Journal of Power Sources 110, (2001), 321. 